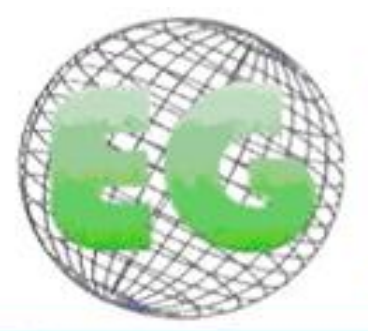

$N^{\circ} 31$

\title{
CLÍNICA
}

\section{Terapia antirretroviral del AIDS en adultos mayores de 50 años: prevalencia y clasificación de los no adherentes}

Terapia antirretroviral da AIDS em adultos acima de 50 anos: prevalência e classificação de não aderenteserapia

AIDS Antiviral Therapy in adults over 50 years old: non adherents' prevalence and classification

\section{*De Mello Padoin, Stela Maris, *Cardoso de Paula, Cristiane, **Spiegelberg Zuge, Samuel, "*Ferreira Langendorf, Tassiane, ${ }^{* * *}$ Pacheco dos Santos, Érika Eberline, ${ }^{* *}$ Ribeiro Primeira, Marcelo}

\begin{abstract}
*Doctora en Enfermería. Profesora del Departamento de Enfermería y del Programa de PosGraduación. **Enfermero/a. Mestrando/a del Programa de Pos Graduación. Bolsista Reuni. E-mail: samuelzuge@gmail.com ${ }^{* * *}$ Estudiante de Enfermería. Bolsista de Iniciación Científica.
\end{abstract}

Universidad Federal de Santa Maria (PPGEnf-UFSM/RS). Brasil.

Palabras clave: Salud del anciano; Síndrome de Inmunodeficiencia Adquirida; HIV; Terapia antirretroviral de alta actividad; Adhesión a la medicación; Enfermería.

Palavras-chave: Saúde do idoso; Síndrome da Imunodeficiência Adquirida; HIV; Terapia antirretroviral de alta atividade; Adesão à medicação; Enfermagem

Keywords: Health of the Elderly; Acquired Immunodeficiency Syndrome; HIV; Antiretroviral Therapy, Highly Active; Medication Adherence; Nursing.

\section{RESUMEN}

Objetivo: Analizar la adherencia al terapia antirretroviral, a partir de la determinación de las variables de clasificación de los no adherentes, según las variables de comportamiento, y de la definición de la prevalencia de no adhesión en los adultos mayores de 50 años, atendidos en el Hospital Universitario Santa Maria / RS.

Método: Investigación de enfoque cuantitativo, tipo descriptivo y transversal. La población de estudio estaba formado por pacientes mayores de 50 años que tienen el HIV / AIDS, y que estaban bajo tratamiento en HUSM, por un total de 72 pacientes. La recolección de datos se desarrolló en el periodo de abril de 2009 hasta octubre de 2010. Se realizaron análisis univariado (porcentajes de frecuencia) y 
bivariado, cruzando el resultado (cumplimiento del TARV) y las variables de comportamiento de la clasificación de no adherentes, por medio del test de chi-cuadrado.

Resultados: La población de pacientes no adherentes fue de 21, con una prevalencia de incumplimiento del $29,2 \%$. A partir del análisis bivariado fue posible determinar las variables de comportamiento para la clasificación de no adherentes: por alguna razón no tomó alguna de las dosis, deje de tomar los medicamentos por el consumo de alcohol, los efectos secundarios impidieron tomar alguna dosis del medicamento, no tomó el medicamento durante el tiempo que estaba trabajando.

Conclusiones: Después de identificar la prevalencia de no adhesión al TARV, y la determinación de las variables que determinan la clasificación de los no adherentes, se pudo conocer la población de no adherentes y las variables de comportamiento que indican esta capacidad para la no ingestión del $100 \%$ de las dosis prescritas.

\section{RESUMO}

Objetivo: analisar a aderência a terapia antirretroviral, a partir da determinação das variáveis de classificação de não aderentes, segundo variáveis comportamentais, e da definição da prevalência de não adesão em adultos acima de 50 anos, atendidos no Hospital Universitário de Santa Maria/RS.

Método: pesquisa de abordagem quantitativa, do tipo descritivo e de delineamento transversal. A população do estudo se formou de pacientes com idade acima de 50 anos que têm HIV/aids, e que estavam em tratamento no HUSM, totalizando 72 pacientes. A coleta de dados se desenvolveu no período de abril de 2009 até outubro de 2010. Foram realizadas análises univariada (frequência percentual) e bivariada, cruzando o desfecho (adesão ao TARV) com as variáveis comportamentais de classificação de não aderentes, por meio do teste do qui-quadrado.

Resultados: a população de não aderentes foi de 21 pacientes, com prevalência de não aderência de $29,2 \%$. A partir da análise bivariada foi possível determinar as variáveis comportamentais de classificação de não aderentes: por algum motivo deixou de tomar alguma das doses; deixou de tomar os medicamentos por uso de bebida alcoólica; os efeitos colaterais impediram de tomar alguma dose do medicamento; não tomou o medicamento durante o horário em que estava trabalhando.

Conclusões: após definir a prevalência da não adesão ao TARV e a determinação das variáveis de classificação de não aderentes, foi possível conhecer a população de não aderentes e as variáveis comportamentais que indicam esta potencialidade para não ingestão dos $100 \%$ das doses prescritas.

\section{ABSTRACT}

Objective: To analyze the antiretroviral therapy adherence, from the determination of non-adherents' classification variables, according behavior variables, and over 50 year old adults' non-adherence prevalence definition, treated at Santa Maria University Hospital (SMUH), RS.

Method: This research presents a quantitative approach, descriptive type and cross-sectional design. This study's population was patients with age above 50 years old that have aids that were treated in $\mathrm{SMUH}$, total of 72 patients. Data collection was developed from April 2009 to October 2010. We performed univariate (frequency percentages) and bivariate analysis, crossing the outcome (ART adherence) and behavioral variables for classification of non-adherents through the chi-square.

Results: Non-adherents' population was 21 patients, with non-adherence prevalence of $29,2 \%$. From bivariate analysis, it was possible to determinate non-adherents' behavior variables classification: for some reason didn't take any of the doses; stopped taking medicines for alcoholic use; side effects prevented from taking any of the medicine doses; didn't take this medicine during working hours.

Conclusions: After defining ART prevalence and non-adherents' behavior variables classifications determinations, it was possible to know the non adherents' population and behavior variables that indicates this potentiality to non ingestion of $100 \%$ prescribed doses. 


\section{INTRODUCCIÓN}

La infección por el Virus de la Inmunodeficiencia Humana (VIH) y la enfermedad Síndrome de Inmunodeficiencia Adquirida (SIDA) han surgido en el mundo a finales de los años 70. En Brasil, los primeros casos fueron registrados en el comienzo de la década de los 80 del pasado siglo, y hasta el año de 2010 fueran acometidas alrededor de 592.914 personas. Las regiones del país que son más afectadas son la Sureste y Sur, con cerca de $58,0 \%$ y $19,0 \%$ de las notificaciones, respectivamente. ${ }^{1}$

El grupo de edad con mayor incidencia de SIDA en Brasil corresponde a 25-49 años. Sin embargo, hay un aumento en los casos reportados en adultos con edad mayor de 50 años, pasando de 17,5\% en 1997 para la 33,3\% en 2009. Con un total de 60.367 casos reportados en este segmento de la población, de 1982 a junio de $2010{ }^{1}$

Los informes muestran la vulnerabilidad de este segmento de la población a las infecciones de transmisión sexual, entre ellas la infección por el VIH. Durante este periodo, el avance de la ciencia médica y farmacéutica ha proporcionado no sólo la extensión de los adultos sexualmente activos com edad mayor de 50 años, sino también la condición de enfermedad crónica, en especial debido a la evolución terapéutica. ${ }^{2,3}$

Después del inicio del tratamiento antirretroviral (TARV), hubo una reducción en la morbimortalidad entre las personas diagnosticadas con SIDA y el número de ingresos hospitalarios, además del incremento en la perspectiva de vida. ${ }^{4}$ Sin embargo, la adhesión al TARV es uno de los más grandes retos para el éxito del tratamiento del VIH/SIDA. ${ }^{5}$

La adherencia al TARV puede ser definida como la concordancia entre la prescripción médica y el comportamiento de la persona en la ingestión de las medicinas. ${ }^{6}$ Esto remite a las implicaciones en el cotidiano farmacológico y en la adherencia al tratamiento. ${ }^{7}$ Así, de esta manera, la eficacia del TARV sufre influencia en los niveles de adhesión, los cuales son considerados determinados para la respuesta terapéutica.

La adhesión representa un desafío para los profesionales que atienden a las personas que tienen VIH / SIDA, por ser preciso articular las dimensiones clínicasinmunológicas y sociocultural. Antes está la necesidad de una evaluación continua del cotidiano de medicación y el cuidado de la salud. En un contexto multiprofesional, es importante conocer las causas de los abandonos de seguimiento de la salud o la frecuencia irregular en el servicio, así como las lagunas en la continuidad de TARV. ${ }^{8}$

Por lo tanto, este estudio tuvo como objetivo analizar la adherencia del TARV en adultos con edad mayor de 50 años atendidos en el Hospital Universitario de Santa Maria / RS, a partir: 1) determinación de las variables para la clasificación de no adherencia, y 2) de definición de la prevalencia de no adhesión.

\section{METODOLOGÍA}

Este estudio presenta un enfoque cuantitativo, descriptivo y transversal. Se trata de un subproyecto de la investigación titulada: Indicadores de vulnerabilidad para adultos y anciano en adhesión al tratamiento antirretroviral en los servicios de referencia en la mitad al sur del Rio Grande do Sul, contemplado en los programas de bolsa de 
iniciación científica, mediante al fomento de la Universidad Federal de Santa Maria UFSM, CNPq y FAPERGS.

El campo de recopilación de datos fue el ambulatorio de enfermedades infecciosas del Hospital Universitario de Santa Maria (HUSM) de Río Grande do Sul, Brasil. La población de estudio estuvo formada por pacientes con edad mayor de 50 años con SIDA y que recibían tratamiento en HUSM.

Los criterios de inclusión fueron: edad mayor de 50 años, mantener acompañamiento en ambulatorio de lo HUSM más de un año, estar registrado en la unidad dispensadora de medicamentos (UDM) del servicio y estar en tratamiento antirretroviral más de tres meses. Los criterios de exclusión fueron: la muerte y el impedimento cognitivo en responder a la entrevista. La población de estudio fue de 75 adultos con edad mayor de 50 años, de los cuales 72 aceptaron participar de la investigación.

La recopilación de datos fue desarrollado entre abril de 2009 hasta octubre de 2010 . A través de una entrevista estructurada se utilizó un formulario que incluía: identificación; características sociodemográficas, de comportamiento y de medicación; cotidiano de medicación (TARV) y de cuidados (actitudes de adhesión); red social primaria y secundaria (apoyo social), relación con el servicio de salud.

Se realizó doble entrada en el administrador Microsoft @ Office Excel $®$ 2007, para composición de la base de datos.

Para el análisis de la adherencia a la terapia antirretroviral, la adhesión se define como el consumo ideal de $100 \%$ de la dosis prescrita, que incluye no sólo la ingesta de medicamentos antirretrovirales (ARV), sino su uso regular, con el fin de disminuir o suprimir carga viral, reduciendo la posibilidad de la aparición de nuevas cepas resistentes virales, lo que compromete el pronóstico del indivíduo. 5, 9

Se realizó un análisis univariado (porcentajes de frecuencia) y el análisis bivariado, cruzando el resultado (la adhesión al TARV) con la clasificación de las variables del comportamiento de adhesión a través de la prueba de chi-cuadrado. El nivel de significación fue igual o inferior a 5\%. Para el procesamiento y análisis estadístico de los resultados se utilizó software Statistical Package for Social Science (SPSS), versión 17.0.

Los aspectos éticos del estudio estaban asegurados, de acuerdo con la Resolución 196/96, y el protocolo de investigación fue aprobado por el Comité de Ética en Investigación (CEP / UFSM) con el número 23081.008437/2007-19.

\section{RESULTADOS Y DISCUSIÓN}

Del total de 72 adultos con edad mayor de 50 años en tratamiento antirretroviral en HUSM / RS, la población de pacientes no adherentes fue de 21. La prevalencia de la no adherentes fue del $29,2 \%$. Este resultado converge con estudios que identificaron la prevalencia de la adhesión y la no adhesión al TARV en personas de diferentes grupo de edades. ${ }^{10,11}$

La población analizada de no adherente, el 80,9\% tenían edad entre 50 y 59 años, $76,2 \%$ eran hombres, el $57,1 \%$ eran blancos, el $61,9 \%$ con educación primaria 
incompleta, el $23,8 \%$ eran casado: $52.4 \%$ tenían una renta fija, el $69,9 \%$ tenía una renta per cápita hasta un salario mínimo, y el 57,1\% informó haber adquirido el VIH por transmisión sexual (Tabla I).

A partir del análisis bivariado fue posible determinar las variables de comportamiento para la clasificación de no adherentes: por alguna razón no tomó ninguna de las dosis; dejó de tomar medicamentos para el consumo de alcohol, los efectos colaterales impidieron tomar alguna dosis de la droga, no tomaron el medicamento durante el tiempo que estaba trabajando (Tabla II), pudiendo cada paciente entrar en más de una variable.

De la población de 21 no adherentes a la TARV, el $57,1 \%$ dejó de tomar cualquier dosis de drogas (la última semana, ayer y anteayer). Las principales razones reportadas fueron: falta de memoria, relación con las actividades cotidianas, el tiempo libre y el ayuno, la ingesta de bebidas alcohólicas, efectos colaterales, tanto clínicos como fisiológicos (necesidad de mantenerse despierto en el trabajo).

Entre los motivos, la falta de memoria es considerada uno de los agravantes que pueden estar asociados con la no aceptación del diagnóstico, además de estar relacionadas con las actividades cotidianas y de tiempo libre. Esta relación puede ser caracterizada como negativa por no seguir una regularidad en los horarios, y por lo tanto, por el no cumplimiento de los horarios prescritos. ${ }^{5}$ 
Tabla I. Características demográficas, sociales y de comportamiento de los pacientes no adherentes. $\mathbf{N}=\mathbf{2 1}$.

\begin{tabular}{|c|c|c|}
\hline \multirow[b]{2}{*}{ Las variables } & \multicolumn{2}{|c|}{ No adherentes } \\
\hline & $\mathrm{N}$ & $\%$ \\
\hline $\begin{array}{r}\text { G rupo de edad } \\
50 \text { a } 59 \\
60 \text { a } 69 \\
70 \text { a } 79\end{array}$ & $\begin{array}{c}17 \\
1 \\
3\end{array}$ & $\begin{array}{c}80,9 \\
4,8 \\
14,3\end{array}$ \\
\hline $\begin{array}{r}\text { Sexo } \\
\\
\text { Femenino } \\
\text { Masculino }\end{array}$ & $\begin{array}{c}5 \\
16 \\
\end{array}$ & $\begin{array}{l}23,8 \\
76,2 \\
\end{array}$ \\
\hline $\begin{array}{ll}\text { Color } & \\
& \text { Blanco } \\
\text { Negro } \\
\text { Pardo }\end{array}$ & $\begin{array}{l}12 \\
5 \\
4\end{array}$ & $\begin{array}{l}57,1 \\
23,8 \\
19,1\end{array}$ \\
\hline $\begin{array}{l}\text { Escolaridad } \\
\text { Educación Primaria Incompleta } \\
\text { Educación Primaria Completa } \\
\text { Educación Secundaria Incompleta } \\
\text { Educación Secundaria Completa } \\
\text { Educación Superior } \\
\text { Incompleta/completa }\end{array}$ & $\begin{array}{c}13 \\
1 \\
3 \\
2 \\
2\end{array}$ & $\begin{array}{l}61,9 \\
4,8 \\
14,3 \\
9,5 \\
9,5\end{array}$ \\
\hline $\begin{array}{l}\text { Estado civil } \\
\text { Casado } \\
\text { Separada /separado } \\
\text { Divorciado } \\
\text { Viudo } \\
\text { Soltero } \\
\text { Vivir juntos } \\
\text { Tiene un novio (a) } \\
\text { Tiene ficante } \\
\end{array}$ & $\begin{array}{l}5 \\
3 \\
3 \\
3 \\
4 \\
1 \\
1 \\
1\end{array}$ & $\begin{array}{c}23,8 \\
14,3 \\
14,3 \\
14,3 \\
19,0 \\
4,8 \\
4,8 \\
4,8 \\
\end{array}$ \\
\hline $\begin{array}{c}\text { Tiene renta fija } \\
\text { Sí } \\
\text { No }\end{array}$ & $\begin{array}{l}11 \\
10\end{array}$ & $\begin{array}{l}52,4 \\
47,6\end{array}$ \\
\hline $\begin{array}{l}\text { Renta per cápita } \\
\text { A uno salario } \\
\text { De uno la dos salarios } \\
\text { Mas de dos salarios }\end{array}$ & $\begin{array}{c}13 \\
3 \\
5\end{array}$ & $\begin{array}{l}61,9 \\
14,3 \\
23,8 \\
\end{array}$ \\
\hline $\begin{array}{l}\text { Forma de transmisión } \\
\text { Transfusión de sangre } \\
\text { Transmisión sexual } \\
\text { Desconocido } \\
\text { Se negó a responder }\end{array}$ & $\begin{array}{c}1 \\
12 \\
7 \\
1\end{array}$ & $\begin{array}{c}4,8 \\
57,1 \\
33,3 \\
4,8\end{array}$ \\
\hline
\end{tabular}

Los que no hacen uso de antirretrovirales por la ingestión de bebidas alcohólicas, representan el $52,3 \%$. El consumo de alcohol es considera un factor que predispone a la falta de adhesión al tratamiento, ${ }^{12,13}$ en Brasil hay un sentido común de que las bebidas y los medicamentos no se pueden mezclar. Esto contribuye a que las personas con buena adhesión suspendan el uso de los medicamentos para consumir alcohol, incluso socialmente. ${ }^{14}$

Tabla II. Variables de clasificación de no adherentes. $\mathbf{N}=\mathbf{2 1 .}$

\begin{tabular}{lccc}
\hline \multicolumn{1}{c}{ Las variables } & $\mathrm{N}$ & No adherentes & \\
\hline $\begin{array}{l}\text { Por alguna razón no tomó ninguna de } \\
\text { las dosis }\end{array}$ & 12 & 57,1 & 0.001 \\
$\begin{array}{l}\text { Dejó de tomar medicamentos para el } \\
\text { consumo de alcohol }\end{array}$ & 11 & 52,4 & 0,048 \\
$\begin{array}{l}\text { Los efectos colaterales impidió de } \\
\text { tomar cualquier dosis de la droga }\end{array}$ & 4 & 19,0 & 0.031 \\
$\begin{array}{l}\text { No tomaron el medicamento durante el } \\
\text { tiempo que estaba trabajando }\end{array}$ & 8 & 38,1 & 0.891 \\
\hline
\end{tabular}


Considerando que el alcohol, culturalmente, forma parte de las reuniones sociales, la imposición de sentido común entre el cuidado de la salud, la adherencia al tratamiento y mantener la vida social activa - participar de las reuniones sociales donde hay oportunidades para el consumo de alcohol - puede provocar conflictos y conducir a la suspensión del tratamiento. Por otra parte, la interacción social influye en la salud y el bienestar de las personas, que se pueden ver limitadas en cuanto a la función social, salud general y la vitalidad presentada por no adherentes la TARV. ${ }^{15}$

La variable "los efectos colaterales impidieron tomar cualquier dosis de la droga" incluye el $19 \%$ de la población. Los informes desfavorable sobre los efectos colaterales de estos medicamentos acaban dejando a los pacientes indispuestos y pueden llevar a interrumpir el tratamiento. ${ }^{16,17}$

Los efectos colaterales causados por los medicamentos es una de las cuatro razones más comunes para la ruptura de la continuidad del tratamiento, así como la falta de memoria, estar lejos de casa y ocupado en las horas correspondientes a ingesta. ${ }^{15}$

Aunque el avance terapéutico proporciona la condición de la enfermedad crónica y la longevidad de las personas que tienen SIDA, también hay que considerar las otras consecuencias de tal avance en la vida diaria de estas personas. Esto teniendo en cuenta que una mayor adhesión al tratamiento puede implicar la potenciación de los efectos colaterales y la fragilidad de los lazos sociales y emocionales. ${ }^{18,19}$

Con respecto a ingerir medicamentos durante las horas de trabajo, el $57,1 \%$ de los pacientes estaban trabajando fuera del domicilio, de los cuales 38,1\% no cumplió con el $100 \%$ de las dosis debidas a esta causa. Tener dosis para ser ingeridas durante las horas de trabajo es considerada una condición de adversidad, pues puede, además de revelar el diagnóstico, generar discriminación. ${ }^{5}$

Para aquellos pacientes cuyo diagnóstico no fue revelado en el lugar de trabajo y que necesitan tomar medicamentos durante este período, estas adversidades aumentan, toda vez que algunos ARVs requieren un cuidado especial, como mantenerlos refrigerados. Cuando se utiliza un refrigerador de uso común, la posibilidad de revelar el diagnóstico a las personas en el trabajo aumenta, y por lo tanto situaciones como éstas terminan activando un impacto negativo en la adhesión al tratamiento. ${ }^{16}$

\section{CONSIDERACIONES FINALES}

Después de definir la prevalencia de la falta de adhesión al TARV, se observó un número considerable de adultos con edad mayor de 50 años que no había mantenido el tratamiento de la ingestión de $100 \%$ de las dosis, según las indicaciones. Esto demuestra la importancia de este hallazgo para la salud individual, asociado a la posibilidad de transmisión del VIH y la morbilidad por SIDA.

La determinación de las variables para la clasificación de no adherentes ayudó a entender las variables de comportamiento que indican el potencial de esta población no ingerir $100 \%$ de las dosis según las indicaciones. Dejar de tomar algunas de las dosis de la droga reveló que las personas tienen diferentes razones, entre las que se destacó la falta de memoria. La influencia del consumo de alcohol en la adhesión fue independiente de la cantidad que la persona bebió. Los efectos colaterales siguen impidiendo tomar cualquier dosis del medicamento, incluso con el avance de la tecnología que permite ajustes en el régimen de medicación. Tomar el medicamento 
durante las horas de trabajo también pone de manifiesto la necesidad de adaptarse a la prescripción diaria de cada persona.

Por lo tanto, hay una necesidad de ampliar la discusión sobre el tema, con el fin de evaluar el control de la salud de las personas que tienen el VIH / SIDA. Es esencial tener en cuenta el examen de su composición, de acuerdo con las exigencias de la vida diaria, así como la educación para la promoción de la salud y prevención de enfermedades, mantenimiento y recuperación de la salud de esta población.

Así pues, es necesario invertir en un modelo de atención multiprofisional que considere las necesidades individuales y colectivas de este segmento de la población, el aumento de la adhesión a la terapia antirretroviral y, en consecuencia, lo que permite la reducción de la morbilidad y mortalidad y mejorar la atención de salud.

\section{REFERENCIAS}

1. Brasil, Ministério da Saúde. Programa Nacional DST/AIDS. Boletim Epidemiológico Aids/DST.[citado 2010 jan]. Disponível em: URL: http://www.aids.gov.br/sites/ default/files/anexos/publicacao/2010/45974/boletim_2010_pdf_29881.pdf [2011 jul 1].

2. Lombardo Pereira G, Costa Aguiar BG. Envelhecendo com Aids ou a Aids no envelhecimento: perfil epidemiológico em um Hospital Universitário do Rio de Janeiro. 2012 abr; 11(2): 21-31.

3. Polejack L, Seidl EMF. Monitoramento e avaliação da adesão ao tratamento antirretroviral para HIV/Aids: desafios e possibilidades. Ciência \& Saúde Coletiva 2010; 15(supl. 10) p. 1201-8.

4. Brasil. Ministério da Saúde. Boletim Epidemiológico Aids. Projeto de vigilância sentinela do HIV: uma apreciação da amostragem e dos resultados obtidos no período de 1997 - 1999 em serviços de DST e prontos-socorrosa. 2000.

5. Silva NLCN, Waidman MAP, Marcon SS. Adesão e não-adesão à terapia antiretroviral: as duas faces de uma mesma vivência. Rev Bras Enferm 2009; 62(2): 21320.

6. Malassiotis A. Lopes V. Chung WY. Factors as sociated with adherence to antiretroviral medication in HIV 1 infected patients. Int J STD \& AIDS 2002; 13: 301 310.

7. Padoin SMM, Machiesqui SR, Paula CC, Tronco CS, Marchi MC. Cotidiano terapêutico de adultos portadores da síndrome de imunodeficiência adquirida. Rev enferm UERJ 2010; 18(3): 389-93.

8. Brasil, Ministério da Saúde, Secretaria de Vigilância em Saúde. Programa Nacional de DST e Aids. Manual de adesão ao tratamento para pessoas vivendo com HIV e Aids. 2008.

9. Barroso LMMB. Adesão ao tratamento com antirretrovirais entre pacientes com Aids. Online Bras J Nurs [periódico online] 2006; Disponível em http://www. uff.br/objnursing/index.php/nursing [2010 fev 1].

10. Colombrini MRC, Coleta MFD, Lopes MHBM. Fatores de risco para a não adesão ao tratamento com terapia antirretroviral altamente eficaz. Rev Esc Enferm USP 2008; 42(3): 490-5.

11. Carvalho CV, Merchab-Hamann E, Matsushita R. Determinantes da adesão ao tratamento antirretroviral em Brasília, DF: um estudo de caso-controle. Rev Soc Bras Med Trop 2007; 40(5): 555-565.

12. Rego SEM, Rego DMS. Associação entre uso de álcool em indivíduos com AIDS e adesão ao tratamento antirretroviral: uma revisão de literatura. J Bras Psiquiatr 2010; 59(1):70-73. 
13- Rego SEM, Oliveira CFA, Rego DMS, Júnior RFS, Silva VB. Estudo do autorrelato de adesão e uso problemático de indivíduos com aids em uso de HAART. J Bras Psiquiatr. 2011; 60(1): 46-49.

14. Melchior R, Nemes MIB, Alencar TMD, Buchalla CM. Desafios da adesão ao tratamento de pessoas vivendo com HIV/Aids no Brasil. Rev Saúde Pública 2007; 41(supl. 2): 87-93.

15. Wang H, Zhou J, He G, Luo Y, Li X, Yang A et al. Consistent ART Adherence Is Associated with Improved Quality of Life, CD4 Counts, and Reduced Hospital Costs in Central China. AIDS research and human retroviruses 2009; 25(8): 757-63.

16. Colombrini MRC, Lopes MHBM, Figueiredo RM. Adesão à terapia antiretroviral para HIV/aids. Rev Esc Enferm USP 2006; 40(4): 576-81.

17. Guerra CPP, Seidl EMF. Adesão em HIV/Aids: estudo com adolescentes e seus cuidadores primários. Psicologia em Estudo 2010; 15(4): 781-789.

18. Geocze L, Mucci S, Marco MA, Martins LAN, Citero VA. Qualidade de vida e adesão ao tratamento antirretroviral de pacientes portadores de HIV. Rev Saúde Pública 2010; 44(4): 743-9.

19. Reis AC, Lencastre L, Guerra MP, Remor E. Relação entre Sintomatologia Psicopatológica, Adesão ao Tratamento e Qualidade de Vida na Infecção HIV e Aids. Psicologia: Reflexão e Crítica 2010; 23(3): 420-429. 